\title{
Phronesis
}

\section{Les identités professionnelles dans le miroir du discours}

Concepts, méthodes et valorisation des rôles

\section{The professional identities Throught the lens of discourse}

Concepts, methods and valorization of specific roles concepts, methods and valorization of specific roles

\section{Jean-Claude Kalubi}

Volume 3, numéro 3, été 2014

Les identités professionnelles dans le miroir du discours

URI : https://id.erudit.org/iderudit/1026389ar

DOI : https://doi.org/10.7202/1026389ar

Aller au sommaire du numéro

Éditeur(s)

Université de Sherbrooke

ISSN

1925-4873 (numérique)

Découvrir la revue

Citer ce document

Kalubi, J.-C. (2014). Les identités professionnelles dans le miroir du discours :

concepts, méthodes et valorisation des rôles. Phronesis, 3(3), 1-4.

https://doi.org/10.7202/1026389ar 


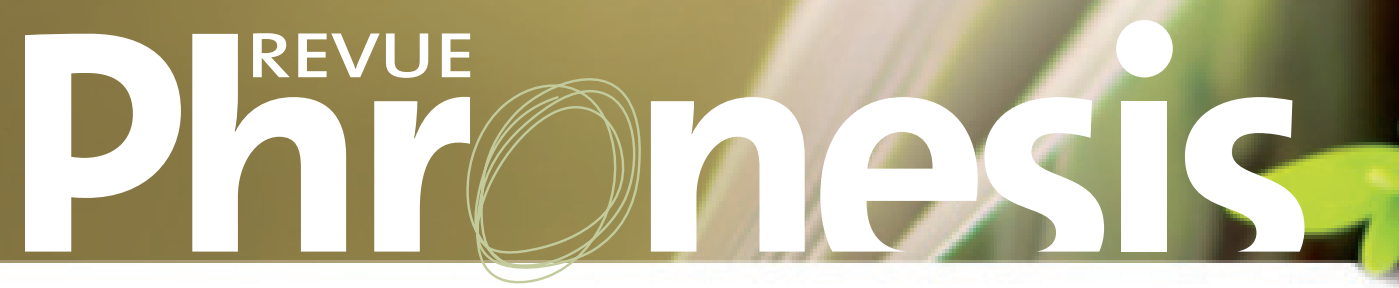

Les identités professionnelles dans le miroir du discours

\section{Jean-Claude KALUBI}

Chaire de recherche sur les identités et les innovations professionnelles en déficience intellectuelle et en troubles envahissants du développement

Université de Sherbrooke

2500 boul. de l'université

Sherbrooke, Québec, Canada, J1K 2R1

Concepts, méthodes et valorisation des rôles 


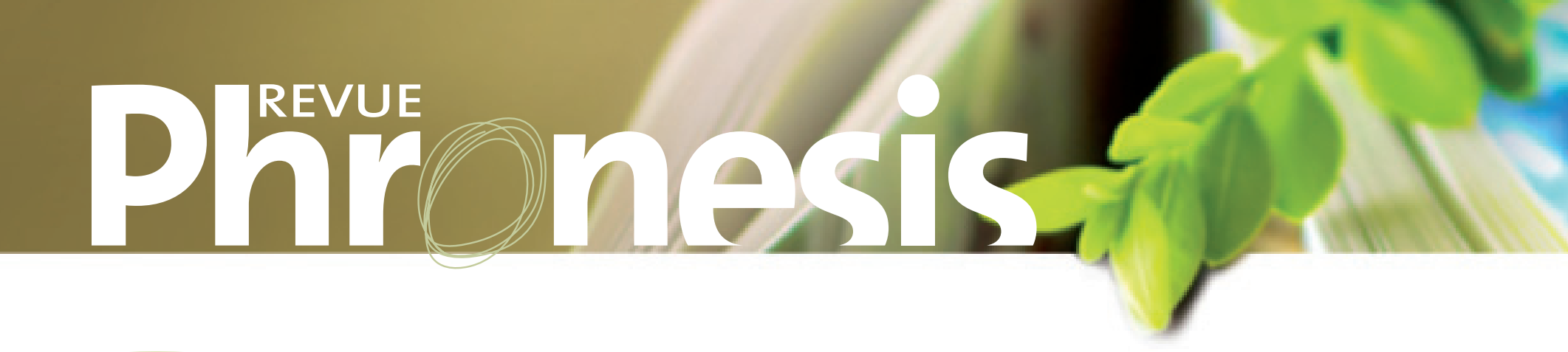

Depuis quelques décennies, le discours académique présente l'identité professionnelle comme étant d'abord un construit social. Il met en évidence des phases de socialisation vécues en milieu professionnel et explore les tendances de développement permettant aux acteurs de se maintenir ou de se renforcer. Le discours relatif aux identités professionnelles suscite des réflexions concernant les représentations, les savoirs, les compétences, les valeurs et les attitudes. Il traite aussi des transformations des relations de travail. Il offre des pistes d'analyse en rapport avec les mouvements d'émancipation, de revendication ou de quête de reconnaissance. Il énonce une série d'exigence en matière de respect de la dignité des travailleurs. Dans d'autres contextes, ce discours aborde les difficultés de certains professionnels à se définir, à présenter leurs caractéristiques personnelles et à maintenir leurs spécificités (Varghese, Morgan, Johnston \& Johnson, 2005). Il témoigne des incertitudes relevées sur le terrain.

Plusieurs études ont récemment rappelé l'importance du discours des identités professionnelles. Ainsi, comme le montre Sachs (2001, 2005), dans ses écrits consacrés à l'identité professionnelle de l'enseignant, le discours relève d'une construction visant à mettre en évidence les conditions de changement, les aspirations individuelles et les préoccupations professionnelles. Il permet de découvrir les tensions entre acteurs autour des injonctions administratives, des ajustements structurels, ainsi que des aspirations démocratiques.

En partant des rapports journaliers des médecins stagiaires, des chercheurs (Apker et Eggly, 2004; Jarvis-Selinger, Pratt \& Regehr, 2012) parlent du poids de l'idéologie dans la construction d'une nouvelle identité professionnelle. Ils mettent en évidence des stratégies de reproduction d'un système élitiste. Ils soulignent aussi l'existence des tensions entre deux modèles de développement professionnel. Le premier est traditionaliste; il valorise les approches humanistes et socialisantes. Le second est suprémaciste; il prône une affirmation identitaire accentuée et l'expansion des privilèges socioéconomiques.

Dans le domaine de la formation en sciences infirmières, les thèmes ci-dessus ont été relevés dans le discours éditorial de la revue professionnelle L'infirmière du Québec. Le discours des éditorialistes (1993-2003) planche sur les conditions de travail; il traite des relations entre l'élite professionnelle et les infirmières de chevet. D’après Nazon (2015), ce discours est discriminatoire au détriment de la majorité des infirmières, dont il occulte les problèmes quotidiens. Il est plutôt orienté vers la promotion de la vision scientifique des soins infirmiers permettant de répondre non seulement aux critères de modernité, d'excellence et d'innovation, mais aussi aux qualités de responsabilité, de planification et de gestion favorable aux politiques de virage ambulatoire. Plusieurs dimensions de l'identité sont évacuées des éditoriaux. Il en est ainsi des stratégies d'insertion des jeunes infirmières, de leur appartenance à ce domaine, de leur adaptation aux changements sociétaux ou à l'évolution de la population. Tout cela est mis en berne au profit d'une expression politique et d'un renforcement de l'affirmation collective. Dans le domaine de la déficience intellectuelle et des troubles du spectre de l'autisme, des intervenants venant de plusieurs régions du Québec (Kalubi, 2011) ont été consultés dans le cadre des sessions de leurs conseils multidisciplinaires. Leurs préoccupations touchaient la transformation des services offerts. Le discours consigné témoigne de plusieurs questions et réponses, renvoyant à la transformation des conditions de travail, à l'évolution des caractéristiques des personnes, ainsi qu'aux défis propres à leurs identités professionnelles. Il étale des réflexions formulées au cours des rencontres. Il montre que le quotidien des intervenants se veut un laboratoire clinique et en même temps un contexte propice au développement des compétences professionnelles. Le discours de ces intervenants attire aussi l'attention sur des facilitateurs et obstacles en lien avec le processus de transformation de l'offre de services. Par ailleurs, la diversité des catégories de représentants (éducateurs spécialisés, psychologues, ergothérapeutes, psychoéducateurs, travailleurs sociaux, infirmiers, etc.) au sein de nombre des conseils multidisciplinaires a permis de rendre compte du foisonnement des discours et de l'importance de la mobilisation sur le terrain. Elle a permis aussi de comprendre le sens des affirmations identitaires.

La notion de discours ressort de la plupart des travaux ci-dessus comme une somme de caractéristiques en rapport avec une pratique en évolution, une production déterminée soumise à l'influence de l'histoire, de la culture, des croyances ou des systèmes de représentations sociales (Maingueneau, 2005).

Le discours des identités professionnelles émerge surtout comme une production en constante évolution. Il traduit la volonté de tout travailleur de voir s'intégrer ses divers rôles, statuts et expériences. Il comprend nombre d'énoncés qui aident à faire ressortir une image de soi cohérente et significative (Castiglione, 2009; Eisenstadt \& Giesen, 1995; Epstein, 1978). Il apparaît alors comme le témoin privilégié des expériences partagées, des apprentissages situés et de la participation communautaire. Il laisse découvrir aussi ce que le professionnel choisit de savoir, de partager et d'introduire dans ses interactions avec les membres de ses communautés professionnelles (Wenger, 1998). Il est pardessus tout une des voies d'illustration de la participation sociale inclusive.

Il permet d'expliciter des facteurs de risque et facteurs de protection accompagnant les objectifs d'un devenir professionnel ou entourant les trajectoires d'ascension personnelle. Plusieurs de ces facteurs renvoient aux routines professionnelles, aux niveaux d'expertise et de qualification, aux activités de formation continue, aux types d'agir communicationnel (Habermas, 1987), aux incitatifs et valeurs communes, ainsi 
qu'à l'homogénéité des tâches. Les dimensions du discours employé par différents professionnels rappellent non seulement la diversité des formes d'engagement, mais aussi la multiplicité des sources d'identité (Brewer, 2001; Wenger, 1998).

Au-delà des statuts et rôles mis en lumière, le discours des identités professionnelles n'impose sa pertinence que lorsqu'il permet d'intégrer de nouveaux apprentissages, de nouvelles connexions, des connaissances spécifiques, ainsi que des expériences de transformation au sein d'une communauté d'apprentissage. D’autres particularités du discours méritent dêtre soulignées. Il en est ainsi de la récurrence des crises et difficultés en milieu professionnel, au cours des interactions professionnelles, ou dans différentes structures socioculturelles et organisationnelles. Il s'agit souvent de préciser la vision de l'avenir, les codes de gestion, les canaux de communication et les modes d'interaction entre acteurs.

Il convient de rappeler les principes et théories du discours. Ce dernier doit être considéré comme une production communicationnelle, comme une structure transphrastique orientée et active. Dans l'optique de Van Djick (1985), il se construit autour d'un ensemble d'énoncés effectifs, dans des situations d'interaction. Il intègre la diversité des langages, des thèmes, des rôles, des sources et des configurations spatiotemporelles (Maingueneau, 2005). En ouvrant sur des effets de conjoncture et sur quelques événements spécifiques, il aide à illustrer des liens entre l'expression des acteurs et leurs entités sociales de référence, dans des contextes de recherche de survie, de reconnaissance, d'hégémonie ou d'alliance. Il ouvre la porte à la découverte des antagonismes et déploiements stratégiques entre certaines forces en présence. L'accent mis sur les conditions de production et les défis sociaux donne au discours une valeur inestimable d'archive, au sens de Foucault, dans la complexité de ses liens avec l'histoire du groupe professionnel concerné. Il permet d'en comprendre la culture du changement, grâce aux énoncés, aux événements ou aux choses qui apparaissent, disparaissent, s’effacent ou réapparaissent.

L'univers du discours des identités professionnelles rend compte de l'évolution des représentations professionnelles, des compétences interprofessionnelles, des qualifications multidisciplinaires et des affiliations institutionnelles (Martineau \& Presseau, 2004; Portelance, Martineau, Presseau \& Rojo, 2012). Comme lieu de reproduction de la parole et de la pensée des professionnels, ce discours est porteur de connaissance en rapport avec les défis spécifiques de l'aide à la personne et à sa famille, dans un contexte de transformation des responsabilités populationnelles. Il renvoie à des images et symboles typiques liés à des situations organisationnelles ou des situations de dénonciation; il se fait le creuset des mouvements créateurs de la transformation des consciences de groupe.

Il emprunte de multiples voies d'actualisation, aussi bien dans sa forme (marquage significatif, style, repères, vocabulaire, etc.) que dans son contenu (informations, directives, messages, consignes, plans d'intervention, projets, etc.). Il offre des ouvertures en rapport avec les processus décisionnels et fonctionnels sur le terrain d'intervention. Or, chaque discours peut mettre en évidence des jeux de présence-absence, des défis disciplinaires, des spécificités subjectives, des représentations individuelles et collectives, des tensions particulières, etc.

Le discours des identités professionnelles apparaît comme étant à la fois le lieu de convergence des solitudes, de transformation des récits, de transition entre mondes et de transfert des significations. Sa portée peut être en même temps communicationnelle, interactionnelle et sociale, témoignant d'une évolution vers les finalités de chaque offre de services.

En définitive, l'expression miroir $d u$ discours permet de restituer le caractère pluriel des expériences vécues par les uns et les autres, la diversité des significations, les traits communs entres les définitions de l'identité professionnelle, les attributs identitaires, etc. Elle alimente des explications autour de trois caractéristiques majeures : le raisonnement identitaire, l'équivocité et le primat de la parole. Dans le premier cas, il s'agit de comprendre comment les mouvements créateurs du discours sont inséparables des circonstances réelles d'existence dans lesquelles évoluent les différents acteurs professionnels. Le raisonnement identitaire émergeant de ce contexte se construit et s'accompagne de la force des stéréotypes, des palimpsestes, des illusions, des mimétismes, des représentations et des expressions simples traduisant les préoccupations d'identité professionnelle.

Par ailleurs, différents témoignages montrent que le discours des identités professionnelles est souvent chargé d'équivocité. Des formules magiques ou passepartouts que répètent les employés ne peuvent pas suffire pour raturer l'écart entre les aspects d'identité vus comme réels et ceux qui sont réclamés, souhaités, professés ou déclarés. Même quand il est ancré dans l'expression des sentiments négatifs et dans l'expression des protestations ou des désapprobations, ce discours tend à encourager des perspectives mélioratives, laudatives et efficientes. Par rapport au primat de la parole, le discours des identités professionnelles est fait de beaucoup d'actes d'affirmation et d'autodéfense. Or, selon certains métiers, les paroles peuvent être considérées comme des actions ou des formes d'action (Kerbrat-Oreccioni, 2001). Revendiquées par tous les professionnels, elles deviennent des outils naturels ou essentiels dans les interactions quotidiennes pour accompagner aussi bien les interventions, les gestes, les pratiques de gestion et les actes thérapeutiques visant à provoquer chez l'usager un changement ou une réaction bénéfique. Dans le contexte de transformation des pratiques, la parole devient « une arme », un régulateur important dans l'expression des comportements.

En définitive, le discours n'est ni neutre ni homogène; il peut renvoyer tant aux traditions scientifiques et culturelles, qu'aux intérêts de différentes sciences humaines, aux options des leaders charismatiques, aux corpus médiatiques et politiques, aux visées de libération, aux revendications institutionnelles, etc.

Le présent numéro thématique porte sur le discours relatif aux identités professionnelles. Il présente des travaux empiriques et des réflexions en sciences humaines et sociales. En plus d'aider à découvrir la complexité des pratiques décrites, il tente d'apporter des réponses aux questions ciblant entre autres le fonctionnement des systèmes, la transformation des services et le développement professionnel.

L'article de Sophie Baconnet offre des éléments pour une excellente entrée en matière; il prend appui sur le discours exprimé en situation de stage d'enseignement, pour cerner les conditions de mobilisation des savoirs professionnels. Il souligne l'ambiguité des règles d'action qui 
orientent le développement professionnel et les expériences pratiques. Il aide à relever dans le discours post activité du stagiaire des indicateurs de professionnalisation et des repères du raisonnement identitaire.

Dans un autre ordre d'idées, Julie Ruel, André Moreau et Johanne April abordent le discours de différents acteurs intersectoriels impliqués dans la transition vers le préscolaire d'enfants ayant des besoins particuliers. Ils traitent des processus structurels et de la dynamique interactionnelle qui se développe entre les acteurs. Ils aident à découvrir la façon dont ces derniers travaillent en réseau, en vue de favoriser la qualité de la transition, le respect des exigences de planification, la continuité éducative entre différents milieux de vie, ainsi que la mise en place des conditions favorisant l'intégration dans le nouvel environnement.

Par ailleurs, l'article de Nancy Granger et Jean-Claude Kalubi porte sur le discours produit à partir de groupes de soutien d'enseignants du secondaire, dans le cadre d'un dispositif d'accompagnement de leur construction identitaire. Ces espaces de parole ont favorisé un climat de partage misant sur le développement d'un raisonnement identitaire, l'adoption des principes d'éducation inclusive et l'adaptation d'une vision commune permettant de mieux guider leurs actions au sein de l'équipe-école.

Dans l'article de Marie-Josée Dumoulin, l'analyse porte sur un discours d'enseignant dont les expériences ont été reconstruites à travers trois logiques transactionnelles. Elle permet de montrer que le processus de restructuration du raisonnement identitaire s'enclenche et se renforce au cours de la première année de pratique. Un tel processus permet de suivre l'implantation d'un dispositif de mentorat utile dans le double registre de la formation et de la recherche.

Pour leur part, Karoline Girard, Jean-Marie Miron et Germain Couture traitent du discours des relations parents-professionnels en contexte d'intervention précoce, autour de la question stratégique du développement du pouvoir d'agir. Placée au cœur du discours des identités professionnelles, cette question contribue au choix d'un cadre conceptuel spécifique, en rapport avec les pratiques d'intervention auprès de la famille. Elle aide à montrer que les expériences habilitantes décrites par les parents, dans leurs relations avec les professionnels, constituent un des facteurs déterminants pour le développement du pouvoir d'agir des familles.

Enfin, dans l'article de Thierry Glarner, l'accent est mis sur le discours et les récits de pratiques des assistants sociaux. Le raisonnement identitaire développé par ces derniers repose sur l'engagement à se mettre au service des populations exclues. La construction de l'identité professionnelle s'avère alors influencée par le regard porté sur le monde, ainsi que par les relations avec la hiérarchie, les liens auprès de la population aidée et le respect de l'environnement institutionnel. Pour se prémunir des situations de souffrance au travail, tout un art d'agir et de faire ressort comme étant nécessaire.

\section{Références bibliographiques}

Apker, J. et Eggly, S. (2004). Communicating professional identity in medical socialization : considering the ideological discourse of morning report. Qualitative Health Research, 14(3), 411-429.

Jarvis-Selinger, S., Pratt, D. D. et Regehr, G. (2012). Competency is not enough: Integrating identity formation into the medical education discourse. Academic Medicine: Journal of the Association of American Medical Colleges, 87(9), 1185-1190.

Epstein, A. (1978). Ethos and Identity. Londres : Tavistock.

Habermas, J. (1987). Théories de l'agir communicationnel. Paris : Fayard.

Brewer, M.B. (2001). The many faces of social identity : Implications for political psychology. Political Psychology, 22(1), 115-125.

Castiglione, D. (2009). Political Identity in a Community of Strangers, in J. Checkel and P. Katzenstein, European Identity (p. 29-51). Cambridge: Cambridge University Press.

Eisenstadt, S. et Giesen, B. (1995). The construction of collective identity. Archives Européennes de Sociologie, 36, 72-102.

Kalubi, J.C. et al (2011). Agir ensemble pour être autrement. Identités professionnelles chez les intervenants en Di-Ted (rapport). Longueuil : Ciipro.

Kerbrat-Orecchioni, C. (2001). Les actes de langage dans le discours: théories et fonctionnement. Paris : Nathan.

Maingueneau, D. (2005). L'analyse du discours et ses frontières. Marges linguistiques, 6, 64-75.

Nazon, E (2015). L'infirmière du Québec: Un discours d'élites professionnelles, 1993-2003. Bulletin canadien d'histoire de la médecine, 32(1). Document téléaccessible à l’adresse <http://www.cbmh.ca/index.php/cbmh/article/view/1637> .

Martineau, S. et Presseau, A. (2004). Analyse exploratoire du discours sur la pratique chez des enseignants d'un CFER. Revue des sciences de l'éducation, 30(3), 631-646.

Portelance, L., Martineau, S., Presseau, A. et Rojo, S. (2012). Naviguer entre l'instruction et la socialisation : discours d'enseignants québécois du secondaire. Phronesis, 1(4), 98-109.

Sachs, J. (2001). Teacher Professional identity: competing discourses, competing outcomes. Journal of Éducation Policy, 16(2), 149-161.

Sachs, J. (2005). Teacher Education and the Development of Professional Identity: Learning to be a Teacher In P. Denicolo and M. Kopf (dir.). Connecting Policy and Practice: Challenges for Teaching and Learning in Schools and Universities (p. 5-21). Londres: Routledge.

Van Djick, T.A. (1985). Cognitive situation models in discourse production. The expression of ethnic situation models in prejudiced stories. In J.P. Forgas (dir.), Language and social situations (p. 61-79). New York, NY: Springer.

Varghese, M., Morgan, B., Johnston, B. et Johnson, K.A (2005). Theorizing Language Teacher Identity: Three Perspectives and Beyond. Journal of Language, Identity \& Éducation, 4(1), 21-44.

Wenger, E. (1998). Communities of practice: learning, meaning and identity. New York, NY : Cambridge University Press. 